\title{
Determination of $K_{\mathrm{m}}$ and $V$ of an enzyme for an unstable substrate and its application to the oxidation of $\boldsymbol{N}^{\tau}$-methylimidazol-3-ylacetaldehyde by aldehyde dehydrogenase
}

\author{
William L. GITOMER* and Keith F. TIPTON \\ Department of Biochemistry, Trinity College, Dublin 2, Ireland
}

(Received 25 January 1983/Accepted 15 February 1983)

\begin{abstract}
The method of Storer \& Cornish-Bowden [(1974) Biochem. J. 141, 205-209] for determining the lag time in coupled enzyme assays was adapted to enable the kinetic parameters of the second (coupling) enzyme for the intermediate to be calculated. The validity and accuracy of this method of progress-curve analysis was established by comparing the $K_{\mathrm{m}}$ value of glucose 6-phosphate dehydrogenase for glucose 6-phosphate generated in situ by the action of glucose phosphate isomerase on fructose 6-phosphate with that determined from initial-rate measurements. The method was applied to the determination of the $K_{\mathrm{m}}$ value of ox liver cytoplasmic aldehyde dehydrogenase for $N^{\tau}$-methylimidazol-3-ylacetaldehyde that was generated in situ by the action of plasma amine oxidase on $N^{\tau}$-methylhistamine.
\end{abstract}

The determination of the $K_{\mathrm{m}}$ and $V$ values for an enzyme-catalysed reaction can prove to be difficult if the substrate is not sufficiently stable to allow accurate determination of its concentrations, and its non-enzymic reactions may give products that could interfere with the reaction under study. Such a case occurred in our studies on the oxidation of $N^{\tau}$ methylimidazol-3-ylacetaldehyde (for an explanation of the nomenclature of these compounds see IUPAC-IUB Commission on Biochemical Nomenclature, 1972) by aldehyde dehydrogenase (EC 1.2.1.3). Since we were unable to prepare a homogeneous preparation of this substrate, an assay method was used for the detection of this activity in which the substrate was generated in situ by the action of plasma amine oxidase (EC 1.4.3.6) on $N^{\tau}$-methylhistamine (Gitomer \& Tipton, 1983).

In the present paper a method, based on the analysis of coupled assay systems (Storer \& Cornish-Bowden, 1974), is described that allows the $K_{\mathrm{m}}$ and $V$ values to be determined from the progress curves in cases where a significant lag period can be observed before a steady-state rate is achieved. The method has been applied to the determination of these values for the glucose 6-phosphate dehydrogenase (EC 1.1.1.49) oxidizing glucose 6-phosphate generated in situ by the action of glucose

\footnotetext{
* Present address: Laboratory of Metabolism, National Institute on Alcohol Abuse and Alcoholism, 12501 Washington Avenue, Rockville, MD 20852, U.S.A.
}

phosphate isomerase (EC 5.3.1.9) on fructose 6-phosphate, and for aldehyde dehydrogenase catalysing the oxidation of $N^{\tau}$-methylimidazol-3ylacetaldehyde generated in situ by the action of plasma amine oxidase on $N^{\tau}$-methylhistamine. In the former case the values calculated by this procedure were found to be in good agreement with those determined from initial-rate measurements.

\section{Materials and methods}

Yeast glucose phosphate isomerase, yeast glucose 6-phosphate dehydrogenase, glucose 6-phosphate, fructose 6-phosphate, $\mathrm{NAD}^{+}, \mathrm{NADP}^{+}$and triethanolamine hydrochloride were obtained from Boehringer Corp., Lewes, East Sussex, U.K. Ox plasma amine oxidase, which was free from any detectable aldehyde-oxidizing activity, was obtained from Sigma Chemical Co., St. Louis, MO, U.S.A. $N^{\tau}$-Methylhistamine was a gift from Smith, Kline and French, Welwyn Garden City, Herts., U.K. All other chemicals were analytical grade or better. $\mathbf{O x}$ liver cytoplasmic aldehyde dehydrogenase was purified by the method of Smith (1979) up to the CM-cellulose step.

Initial rates of the oxidation of glucose 6phosphate by glucose 6-phosphate dehydrogenase were determined at $30^{\circ} \mathrm{C}$ at $\mathrm{pH} 7.5$ in an assay mixture containing, in a total volume of $2.0 \mathrm{ml}$, $1.5 \mu \mathrm{mol}$ of $\mathrm{NADP}^{+}, 96 \mu \mathrm{mol}$ of triethanolamine hydrochloride, $9.6 \mu \mathrm{mol}$ of EDTA, 3.5 munits 
Table 1. Calculation of the maximum velocity for the oxidation of glucose 6-phosphate by glucose 6-phosphate dehydrogenase

Values of $V_{2}$ were calculated from the data for $v_{2}$ (see Table 2) by using the iterative procedure.

$\begin{array}{ccc}\begin{array}{c}t \text { pair } \\ \text { (arbitrary units) }\end{array} & \overbrace{\text { Fig. 1, curve } A} V_{2} \text { (arbitrary units) } \\ 10,20 & 6.2 & 5.3 \\ 10,30 & 6.4 & 4.4 \\ 10,40 & 5.9 & 4.6 \\ 10,50 & 4.9 & 5.8 \\ 20,30 & 6.7 & 3.8 \\ 20,40 & 5.7 & 4.3 \\ 20,50 & 4.6 & 5.9 \\ 30,40 & 5.0 & 5.0 \\ 30,50 & 4.0 & 8.6 \\ 40,50 & 3.3 & 3.7\end{array}$

Table 2. Calculation of $\phi$ and $K_{m}$ for the oxidation of glucose 6-phosphate by glucose 6-phosphate dehydrogenase

Values of $\phi$ were calculated by using eqn. (2). $K_{m}$ was then determined from the relationship given in eqn. (6). The two values for each time were calculated from curves $A$ and $B$ in Fig. 1.

\begin{tabular}{|c|c|c|c|}
\hline $\begin{array}{c}t \\
\text { (arbitrary units) }\end{array}$ & $\begin{array}{c}v_{2} \\
\text { (arbitrary units) }\end{array}$ & $\phi$ & $\begin{array}{c}K_{\mathrm{m}} \\
(\mu \mathrm{M})\end{array}$ \\
\hline 10 & $\begin{array}{l}0.761 \\
0.853\end{array}$ & $\begin{array}{l}0.189 \\
0.218\end{array}$ & $\begin{array}{l}68 \\
59\end{array}$ \\
\hline 20 & $\begin{array}{l}1.31 \\
1.42\end{array}$ & $\begin{array}{l}0.391 \\
0.441\end{array}$ & $\begin{array}{l}65 \\
58\end{array}$ \\
\hline 30 & $\begin{array}{l}1.74 \\
1.76\end{array}$ & $\begin{array}{l}0.612 \\
0.624\end{array}$ & $\begin{array}{l}63 \\
61\end{array}$ \\
\hline 40 & $\begin{array}{l}2.04 \\
2.06\end{array}$ & $\begin{array}{l}0.816 \\
0.832\end{array}$ & $\begin{array}{l}63 \\
62\end{array}$ \\
\hline 50 & $\begin{array}{l}2.19 \\
2.46\end{array}$ & $\begin{array}{l}0.939 \\
1.206\end{array}$ & $\begin{array}{l}68 \\
53\end{array}$ \\
\hline
\end{tabular}

(10ng) of glucose 6-phosphate dehydrogenase (1 unit catalyses the formation of $1 \mu \mathrm{mol}$ of product/ min) and glucose 6-phosphate. The concentration of glucose 6-phosphate was varied in the range 10 $700 \mu \mathrm{M}$, and $K_{\mathrm{m}}$ and $V$ values were determined from direct-linear plots of these data (Eisenthal \& Cornish-Bowden, 1974).

The lag-time analysis for the determination of the $K_{\mathrm{m}}$ of glucose 6-phosphate dehydrogenase for glucose 6-phosphate was performed at $30^{\circ} \mathrm{C}$ at pH 7.5 in an assay mixture containing, in a total volume of $2.0 \mathrm{ml}, 1.5 \mu \mathrm{mol}$ of $\mathrm{NADP}^{+}, 20 \mu \mathrm{mol}$ of
$\mathrm{MgSO}_{4}, 1.96 \mu \mathrm{mol}$ of fructose 6-phosphate, $88 \mu \mathrm{mol}$ of triethanolamine hydrochloride, $8.8 \mu \mathrm{mol}$ of EDTA, 14 munits (40 ng) of glucose 6-phosphate dehydrogenase and 3.5 munits (10ng) of glucose phosphate isomerase. Before the reaction was started by the addition of glucose phosphate isomerase, the assay mixture was incubated at $20^{\circ} \mathrm{C}$ until all of the glucose 6-phosphate that was present in the fructose 6-phosphate preparation had been oxidized. The time course of the reaction was monitored at $340 \mathrm{~nm}$ with a Beckman DU spectrophotometer with a Gilford 240 absorbance converter. Some studies with a Durrum stopped-flow apparatus indicated that there was no particular advantage in its use with lag times of the magnitude used in these studies.

The lag-time analysis for the determination of the $K_{\mathrm{m}}$ of aldehyde dehydrogenase for $N^{\tau}$-methylimidazol-3-ylacetaldehyde was performed at $30^{\circ} \mathrm{C}$ at pH7.4 in a reaction mixture containing, in a total volume of $1.90 \mathrm{ml}, 125 \mathrm{~mm}$-sodium phosphate buffer, $2.6 \mathrm{mM}-N^{\tau}$-methylhistamine, $700 \mu \mathrm{M}-\mathrm{NAD}^{+}$, 47 munits of ox plasma amine oxidase (1 unit will oxidize $1.0 \mu \mathrm{mol}$ of benzylamine to benzaldehyde/ min at $\mathrm{pH} 7.4$ at $30^{\circ} \mathrm{C}$ ) and 9.8 munits of ox liver cytoplasmic aldehyde dehydrogenase (1 unit will reduce $1.0 \mu \mathrm{mol}$ of $\mathrm{NAD}^{+}$to $\mathrm{NADH} / \mathrm{min}$ at $\mathrm{pH} 7.4$ at $30^{\circ} \mathrm{C}$ ). The reaction was started by the addition of methylhistamine, and the time course was monitored at $340 \mathrm{~nm}$.

\section{Principle}

The method is based on the theory that has been developed for studying coupled enzyme systems of the type:

$$
A \stackrel{E_{1}}{\longrightarrow} B \stackrel{E_{2}}{\longrightarrow} C
$$

where $A \rightarrow B$ is the reaction catalysed by enzyme $E_{1}$, and $B \rightarrow C$ is the reaction catalysed by enzyme $E_{2}$.

Such treatments were, however, devised to allow the calculation of the quantity of coupling enzyme $\left(E_{2}\right)$ required to ensure an accurate measurement of the steady-state rate of $E_{1}$. Thus these equations had to be adapted to the coupled assay system in which the kinetic properties of $\mathrm{E}_{2}$ were to be studied.

Storer \& Cornish-Bowden (1974) derived an equation that describes the time course of the coupled enzyme reaction system shown in scheme (1):

$$
t=\phi K_{2} / v_{1}
$$

where $t$ is the elapsed time from the initiation of the reaction, $K_{2}$ is the Michaelis constant of $E_{2}$ for substrate $\mathrm{B}, v_{1}$ is the reaction rate of $\mathrm{E}_{1}$, and $\phi, a$ dimensionless number, is:

$$
\phi=\frac{V_{2} v_{1}}{\left(V_{2}-v_{1}\right)^{2}} \cdot \ln \left(\frac{v_{1}\left(V_{2}-v_{2}\right)}{V_{2}\left(v_{1}-v_{2}\right)}\right)-\frac{v_{1} v_{2}}{\left(V_{2}-v_{2}\right)\left(V_{2}-v_{1}\right)}
$$


where $v_{2}$ is the reaction rate of enzyme $\mathrm{E}_{2}$ at $t$, and $V_{2}$ is the maximum velocity of $E_{2}$.

For any lag time of a time course for a coupled enzyme reaction one can select two sets of data points $v_{2 t}, t_{t}$ and $v_{2,}, t_{j}$. Substituting each set of data points into eqn. (2) gives two equations, which can be solved simultaneously to give:

$$
\frac{t_{i}}{t_{j}}=\frac{\phi_{i}}{\phi_{j}}
$$

Substituting for $\phi_{i}$ and $\phi_{j}$ and simplifying gives:

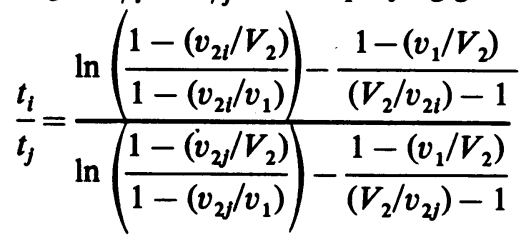

Since $t_{i}, v_{2 l}, t_{j}, v_{2 j}$ and $v_{1}$ can be determined experimentally, one can solve iteratively for $V_{2}$. Once $V_{2}$ is known, one can determine the value of $\phi$ at a given value of $v_{2}$, and then solve for $K_{2}$ by rearranging eqn. (2) to give:

$$
K_{2}=t v_{1} / \phi
$$

Alternatively, using eqn. (2) one can plot $t$ as a function of $\phi$ for various values of $v_{2}, t$ points, and this will give a straight line passing through the origin with a slope of $K_{2} / v_{1}$.

\section{Results and discussion}

Examination of eqn. (5) indicates that there are two experimental requirements that must be met in order for the equation to be solvable. These are: (1) the second enzyme is not operating at its maximal velocity $\left(V_{2}>v_{2}\right)$, and (2) the steady-state velocity of the first enzyme is greater than that of the second enzyme during the period in which measurements are made $\left(v_{1}>v_{2}\right)$. However, both of these requirements must be satisfied if a lag phase is to be observed.

Coupled assays were performed as described in the Materials and methods section. Two such time courses are shown in Fig. 1. When the lag phases were being determined it was essential to ensure an accurate determination of the zero time. Values of $v_{2}$ were obtained by: (1) fitting the experimentally determined lag-phase time course (Fig. 1) to a fourth-order equation, which gave, for example, in the case of curve $A$ :

$$
\begin{aligned}
A=5.317+ & 0.1148 t+3.446 \times 10^{-2} t^{2} \\
& -1.324 \times 10^{-4} t^{3}-7.540 \times 10^{-7} t^{4}
\end{aligned}
$$

by (2) differentiating that equation to obtain an equation of $v_{2}$ as a function of $t$, which gives:

$$
\begin{aligned}
\mathrm{d} A / \mathrm{d} t=v_{2} & =0.1148+6.892 \times 10^{-2} t \\
& -3.972 \times 10^{-4} t^{2}-3.016 \times 10^{-6} t^{3}
\end{aligned}
$$

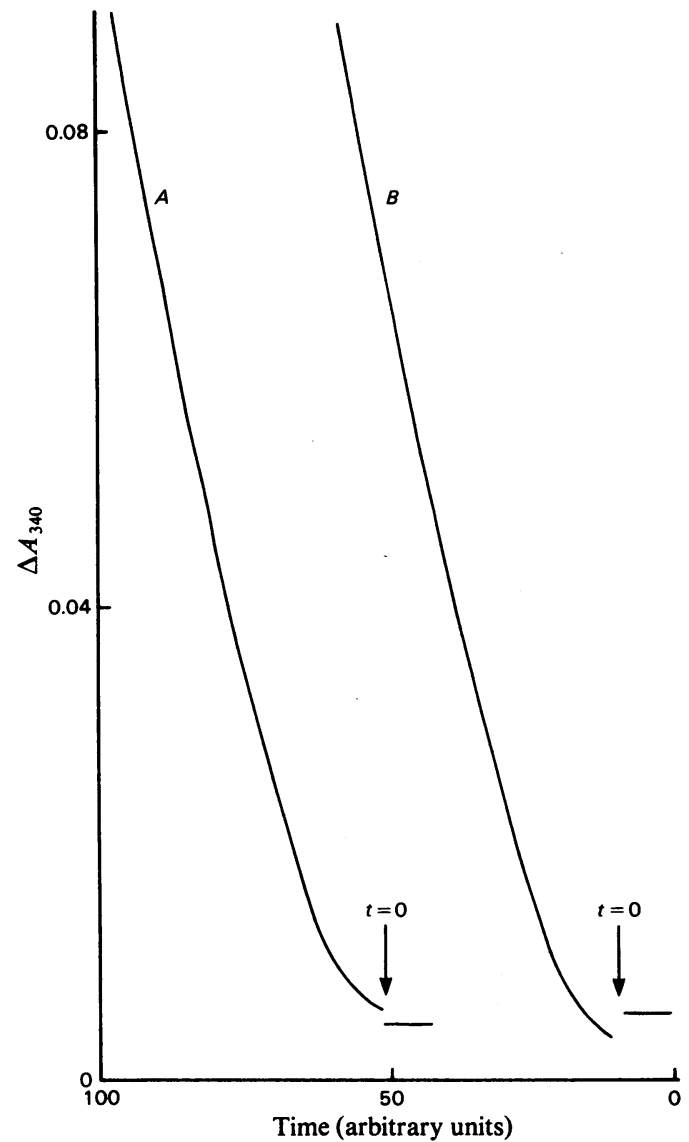

Fig. 1. Time courses of the coupled assays of the glucose phosphate isomerase/glucose 6-phosphate dehydrogenase system that were used to determine the $K_{m}$ value of the second of these two enzymes for glucose 6-phosphate The experimental conditions were as described in the text.

and then by (3) substituting values of $t$ into eqn. (8) to obtain values of $v_{2}$ (see Table 2). Five values of $t$, within the bounds of the data used to obtain eqn. (8), were found to be a convenient number to use for the analysis of each lag phase.

An accurate estimate of $v_{1}$ must be obtained independently before a value for $V_{2}$ can be determined. This was determined by assaying the first enzyme with a large excess of the second enzyme. This value and the calculated values for the $v_{2}, t$ pairs were used to determine $V_{2}$ by using an iterative procedure with a Wang computer. The median value determined was taken as the value of $V_{2}$ (Table 1), and was used with each $v_{2}, t$ pair to calculate values for $\phi$ and $K_{2}$ (Table 2). 
For glucose 6-phosphate dehydrogenase the $K_{\mathrm{m}}$ value for glucose 6-phosphate determined by the lag-phase method was $62 \mu \mathrm{M}$ with a $68 \%$ confidence-limit range of 59-65 $\mu \mathrm{M}$; the value of $K_{\mathrm{m}}$ determined by using the direct linear plot (Eisenthal \& Cornish-Bowden, 1974; Cornish-Bowden \& Eisenthal, 1974) was $49 \mu \mathrm{M}$ with a $68 \%$-confidence-limit range of $43-62 \mu \mathrm{M}$. The maximum velocities determined by the two methods were in similar agreement.

This procedure was also used to analyse the lag phase of the coupled plasma amine oxidase/aldehyde dehydrogenase system with $N^{\tau}$-methylhistamine. A value of $K_{\mathrm{m}}$ of aldehyde dehydrogenase for $N^{\tau}$-methylimidazol-3-ylacetaldehyde of $40 \mu \mathrm{M}$ with a $68 \%$-confidence-limit range of $39-49 \mu \mathrm{M}$ was determined under these conditions, and the maximum velocity $\left(V_{2}\right)$ of the preparation was $120 \%$ of that obtained with acetaldehyde as the substrate.

The agreement between the $K_{\mathrm{m}}$ value of glucose 6-phosphate dehydrogenase for glucose 6-phosphate calculated from the progress curve of the coupled assay with that determined by initial-rate studies indicates that this procedure can be used for the accurate determination of kinetic parameters. Its application to the oxidation of $N^{\tau}$-methylimidazol3-ylacetaldehyde by aldehyde dehydrogenase allowed the $K_{\mathrm{m}}$ value for this substrate, which we had been unable to measure by more conventional means, to be determined. The applicability of this method relies on the intermediate, that is the substrate for the enzyme under study, being sufficiently stable under the assay conditions so that its steady-state concentration is not significantly affected by any non-enzymic reactions. The relatively low steady-state concentrations of the intermediate and the short duration of the experiments should, however, minimize any such reactions, and this method may be more generally applicable to the study of enzymes that act on unstable substrates. In the case of the reaction catalysed by aldehyde dehydrogenase the hydration of the aldehyde substrate, after its release from the amine oxidase, might affect the $K_{\mathrm{m}}$ value determined. However, it is unclear whether the hydrated (gem-diol) or unhydrated form of the aldehyde is the true substrate for this enzyme, and it is possible that aldehyde dehydrogenase may in fact be able to bind and catalyse the oxidation of both forms (Weiner et al., 1982).

We are grateful to the Medical Research Council of Ireland for the support of a research grant and to Dr. Robert Eisenthal for helpful discussion.

\section{References}

Cornish-Bowden, A. \& Eisenthal, R. (1974) Biochem. J. 139, 721-730

Eisenthal, R. \& Cornish-Bowden, A. (1974) Biochem. J. 139, 715-720

Gitomer, W. L. \& Tipton, K. F. (1983) Pharmacol. Biochem. Behav. in the press

IUPAC-IUB Commission on Biochemical Nomenclature (1972) Biochem. J. 126, 773-780

Smith, I. L. (1979) Ph.D. Thesis, University of Cambridge

Storer, A. C. \& Cornish-Bowden, A. (1974) Biochem. J. 141, 205-209

Weiner, H., Freytag, S., Fox, J. M. \& Hu, J. H. J. (1982) in Enzymology of Carbonyl Metabolism: Aldehyde Dehydrogenase and Aldo/Keto Reductase (Weiner, $\mathrm{H}$. \& Wermuth, B., eds.), pp. 91-102, Alan R. Liss, New York 